\title{
Evidências e dúvidas sobre o trata- mento cirúrgico do diabetes tipo 2
}

\section{Evidences and doubts about type 2 diabetes surgical treatment}

\author{
Reginaldo Ceneviva ${ }^{1}$, Melina M. Viegas-Silva², José S. dos Santos ${ }^{3}$, Wilson Salgado Júnior ${ }^{4}$, Orlando Castro e \\ Silva Júnior ${ }^{5}$
}

Palavras-chaves: Diabetes mellitus tipo 2. Resistência à insulina. Obesidade. Cirurgia bariátrica. Cirurgia metabólica.

O maior risco para o desenvolvimento do diabetes mellitus tipo 2 (DM2) é a obesidade, sendo que, de acordo com a Federação Internacional de Diabetes, $80 \%$ dos pacientes com DM2 são obesos ou têm sobrepeso.

A prevalência do diabetes do tipo 2 tem aumentado rapidamente nas últimas décadas em muitos países, sobretudo pela prevalência crescente da obesidade, em proporções epidêmicas. Pela sua gravidade e prevalência crescente, a obesidade e o DM2 correspondem a problema medicossocial importante, constituindo ambos um desafio de saúde pública significante no Brasil e no mundo.

O diabetes mellitus afeta cerca de 150 milhões de pessoas em todo o mundo. Este número pode dobrar em 2025. ${ }^{1}$ O DM2 é o que predomina, presente em 90 a $95 \%$ dos pacientes com diabetes, usualmente associado à intolerância à glicose e a sobrepeso.

No DM2 podem ocorrer distúrbios em múltiplos órgãos, incluindo deficiência pancreática (com distúrbios na função da célula $\beta$ e prejuízo na secre- ção de insulina, e distúrbios na função das células $\beta$ com secreção de glucagon aumentada), redução da captação de glicose pelos músculos esqueléticos, aumento da liberação de glicose do fígado e a redução do efeito das incretinas ${ }^{2}$.

A forte associação do DM2 com a obesidade, sobretudo com o acúmulo de gordura abdominal, está ligada à resistência à insulina periférica e hepática, inflamação e subsequente "lipotoxidade" das células $\beta .{ }^{3} \mathrm{O}$ risco de DM2 aumenta 42 vezes nos homens quando o IMC aumenta de $<23 \mathrm{~kg} / \mathrm{m}^{2}$ para $>35 \mathrm{~kg} / \mathrm{m}^{2}$ e 93 vezes nas mulheres quando o IMC aumenta de $<22 \mathrm{~kg} / \mathrm{m}^{2}$ para $>35 \mathrm{~kg} / \mathrm{m}^{2} .^{3,4}$

O tratamento cirúrgico da obesidade apresentou grandes avanços a partir das últimas décadas do século XX em decorrência dos bons resultados de novas técnicas cirúrgicas na redução do peso e na evolução das co-morbidezes. Numerosos estudos comprovaram a eficácia da cirurgia bariátrica em melhorar e mesmo normalizar os níveis da glicose em pacientes obesos com DM2.
1. Professor titular colaborador sênior da Disciplina de Cirurgia Digestiva do Departamento de Cirurgia e Anatomia da Faculdade de Medicina de Ribeirão Preto da Universidade de São Paulo (FMRP-USP) e coordenador do Centro de Cirurgia Bariátrica.

2. Fisioterapeuta do Centro de Cirurgia Bariátrica do Hospital das Clínicas da FMRP-USP.

3. Professor associado da Disciplina de Cirurgia Digestiva do Departamento de Cirurgia e Anatomia da FMRP-USP.

4. Professor assistente doutor da Disciplina de Cirurgia Digestiva do Departamento de Cirurgia e Anatomia da FMRP-USP e cirurgião do Centro de Cirurgia Bariátrica.

5. Professor titular e chefe da Disciplina de Cirurgia Digestiva do Departamento de Cirurgia e Anatomia daFMRP-USP.
Correspondência:

Departamento de Cirurgia e Anatomia da Faculdade de Medicina de Ribeirão Preto USP HCRP, 9ำ andar Campus Universitário 14048-900 - Ribeirão Preto - SP - Brasil

Artigo recebido em 29/11/2011 Aprovado para publicação em 05/12/2011 
Embora os pacientes com diabetes tenham maior dificuldade em perder peso que os não diabéticos, a perda de peso é essencial no tratamento do DM2, diminui a mortalidade e a morbidez. A mudança no estilo de vida, com dieta e exercícios físicos, associada ou não a medicamentos, resulta em perda de peso usualmente modesto e de pequena duração, portanto, sem atração para os pacientes.

Comparada com esse tratamento conservador, que induz à perda de peso de 5 a $10 \%$, mantida por pouco tempo e, frequentemente, seguida por reganho de peso, a cirurgia bariátrica resulta em redução de 30 a $40 \%$ do peso original ou perda de $50 \%$ do excesso de peso, com controle do DM2 na maior parte dos pacientes.

Os procedimentos bariátricos, de acordo com o mecanismo de perda de peso, são classificados em restritivos, disabsortivos e mistos. As técnicas cirúrgicas atuais podem ser divididas de acordo com o mecanismo de redução de peso em: restritivas que, por reduzir a capacidade do estômago, resultam em menor ingestão de alimentos e saciedade precoce; disabsortivas que, mediante a exclusão de segmento extenso do intestino delgado, resultam em má-absorção dos alimentos; e mistas que têm os componentes restritivo e disabsortivo.

Técnicas tidas classicamente como puramente restritivas são: gastroplastia vertical com bandagem (GVB) e banda gástrica ajustável (BGA). A gastrectomia vertical $(\mathrm{GV})$ tem demonstrado resultados melhores que destas, mas está ainda em fase experimental, sem resultados em longo prazo, e provavelmente não atingirá a grande redução de peso observada após cirurgias mistas. (Figura 1).
Técnicas puramente disabsortivas, como a derivação jejunoileal, foram abandonadas pelas altas taxas de distúrbios nutricionais e metabólicos. Atualmente procedimentos disabsortivos utilizados são as derivações biliopancreáticas (DBP) de Scopinaro e sua variante Marceau-Hess (duodenal switch). A derivação biliopancreática pela técnica de Scopinaro envolve hemigastrectomia distal com anastomose gastroileal em Y de Roux. A ressecção parcial do estômago é responsável pelo componente restritivo transitório e consequente diminuição inicial da ingestão que, geralmente, desaparece com o tempo; persiste o componente disabsortivo, sobretudo para gorduras e hidratos de carbono. (Figura 2).

A técnica mista utilizada e predominantemente restritiva é a derivação gástrica em Y de Roux (DGYR), também chamada operação de Fobi-Capella. A DGYR é a mais aceita universalmente para o tratamento cirúrgico da obesidade grau III; envolve gastroplastia vertical com ou sem anel de contenção (componente restritivo) e derivação gastrojejunal em Y de Roux (componente disabsortivo). (Figura 3).

A DGYR e as DBP alteram ainda os níveis sanguíneos de hormônios gastrointestinais: reduzem a secreção de grelina (hormônio orexígeno) e aumentam a secreção de GLP-1 (peptídeo similar ao glucagon 1) e do PYY (peptídeo YY) que são hormônios anorexígenos. Estes procedimentos conseguem redução grande do peso corporal, sustentada por longo tempo.

Em estudo prospectivo multicêntrico na Suécia, Sjostrom et al. demonstraram que, um ano após DGYR, GVB e BGA, a redução de peso é máxima, respectivamente, de $38 \%, 26 \%$ e $21 \%$, com reganho de peso a seguir e mantendo aos 10 anos de pós-ope-

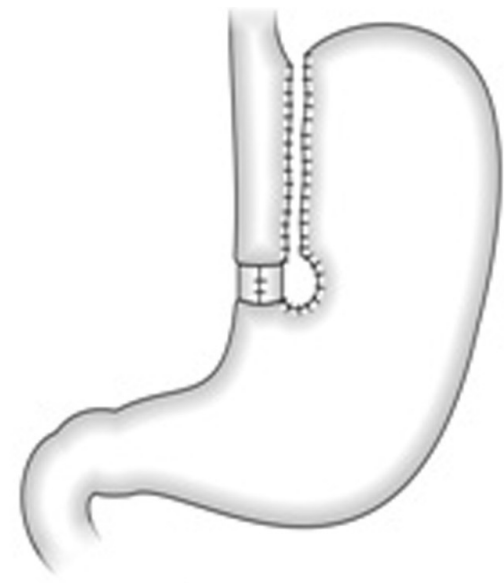

A

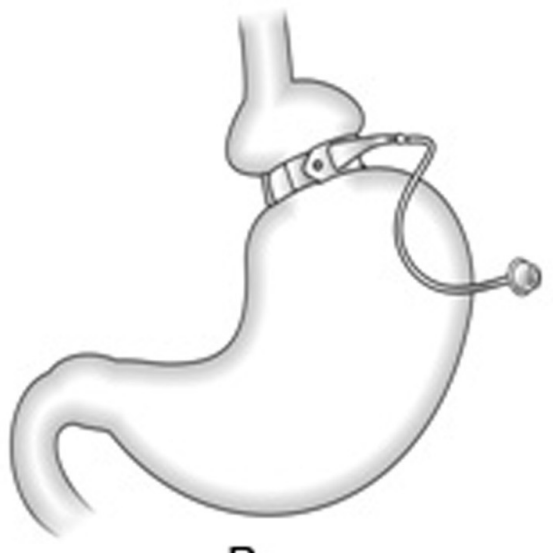

B

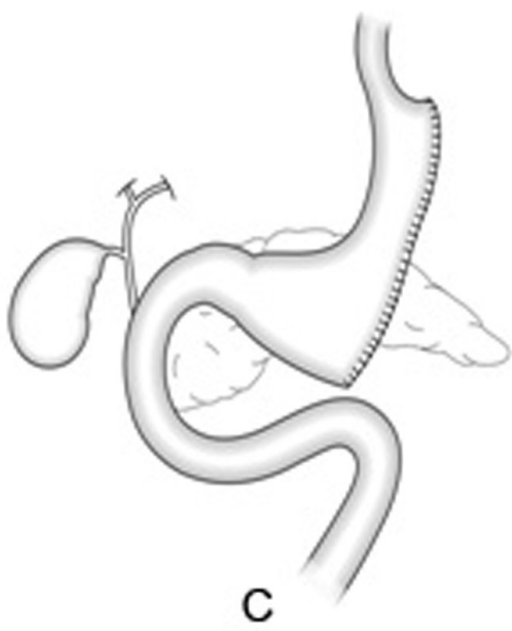

Figura 1. Técnicas restritivas . A) Gastroplastia vertical com bandagem. B) Banda gástrica ajustável. C) Gastrectomia vertical. 

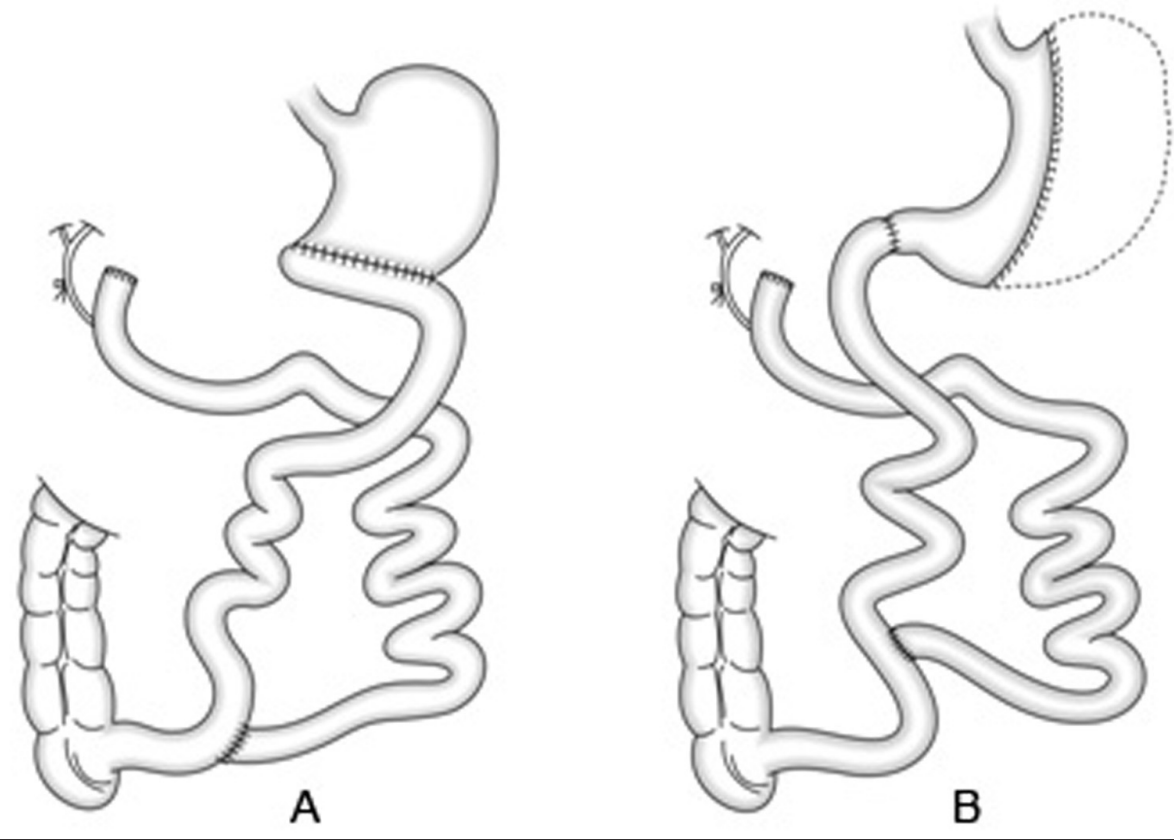

Figura 2. Derivação biliopancreática. A) Scopinaro. B) Duodenal Switch.

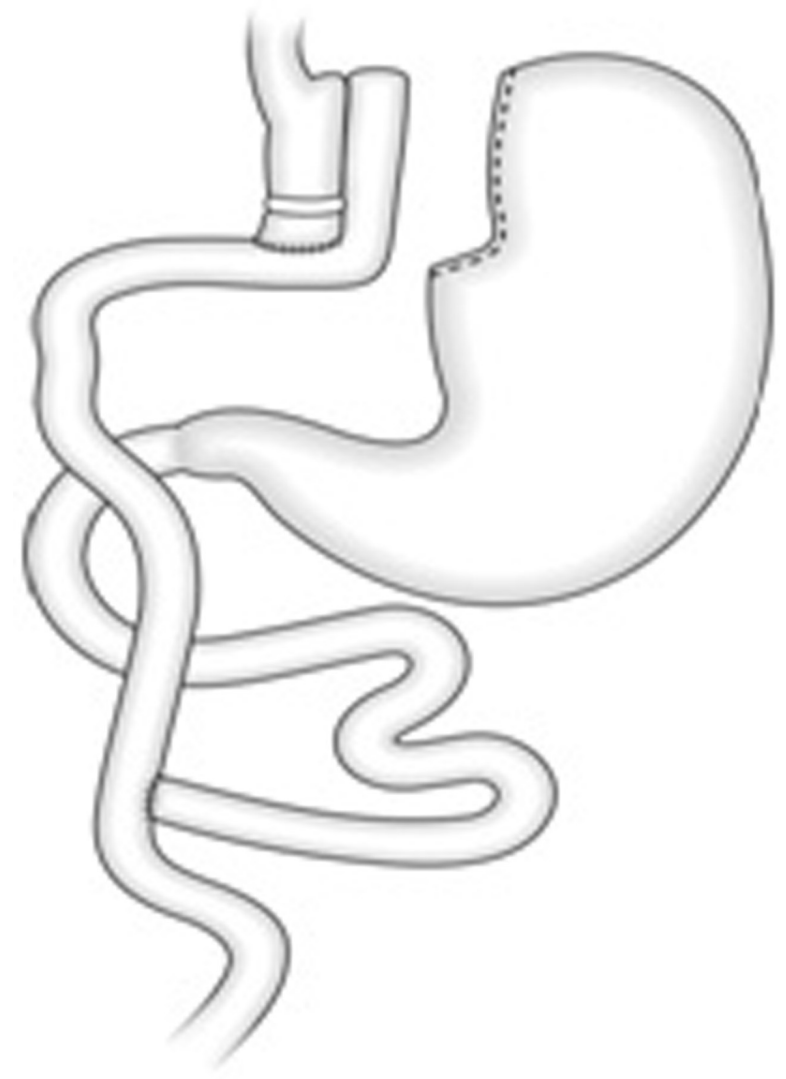

Figura 3. Técnica mista. Derivação gástrica em Y de Roux. ratório redução do peso original, respectivamente, de $25 \%, 16,5 \%$ e $13 \% .{ }^{5}$ A perda de peso original é de 35 a $40 \%$ e a do excesso de peso é de 75 a $80 \% 1$ a 2 anos após as derivações biliopancreáticas.

Operações selecionadas revertem o DM2 completamente em mais de $80 \%$ dos casos $^{6,7}$ por mecanismos que provavelmente vão além da perda de peso.

\section{Cirurgia metabólica}

O conceito de cirurgia metabólica foi definido por Buchwald e Varco em 1978 no seu livro Metabolic Surgery como "o manuseio cirúrgico de um órgão ou sistema normal para conseguir um resultado biológico de melhora na saúde". As operações sobre o estômago e os nervos vagos abdominais (vagotomia) para o tratamento das úlceras pépticas gastroduodenais têm sido associadas à perda de peso e melhora no controle da glicemia em pacientes diabéticos.

Embora alguns relatos tivessem citado melhora do DM2 após cirurgia bariátrica, foram Pories et al. os primeiros a demonstrar e a enfatizar a remissão do diabetes e da intolerância à glicose na maior parte dos pacientes após cirurgia bariátrica em seguimento de longo prazo (14 anos). ${ }^{6}$ Os resultados de meta-análise realizada por Buchwald et al. reforçam esses achados, com alta resolução do DM2 e variações dependentes do tipo de procedimento cirúrgico empregado. Para banda gástrica ajustável, derivação gástrica em 
Y de Roux e derivação biliopancreática, respectivamente, a resolução do diabetes deu-se em $48 \%, 84 \%$ e $98 \% .^{7}$

Os resultados desses e de outros estudos observacionais motivaram cirurgiões, endocrinologistas e outros pesquisadores interessados no tratamento do diabetes. A conferência de Roma em 2007, para avaliar o estado atual e planejar passos futuros no campo da cirurgia metabólica, resultou em algumas recomendações de consenso, das quais as principais foram: ${ }^{8}$

- Modificações anatômicas de várias regiões do trato gastrointestinal provavelmente contribuem para a melhora do DM2 mediante mecanismos fisiológicos distintos.

- Procedimentos de derivação gastrointestinal podem melhorar o diabetes por mecanismos que vão além das modificações na ingestão de alimentos e perda de peso.

- Cirurgias gastrointestinais podem ser apropriadas para o tratamento do DM2 em pacientes que são candidatos cirúrgicos adequados com IMC de 30 a $35 \mathrm{~kg} / \mathrm{m}^{2}$ e que são inadequadamente controlados com estilo de vida e terapêutica médica.

Embora o tratamento cirúrgico do diabetes seja uma indicação para muitos cirurgiões bariátricos, muitos endocrinologistas não têm endossado esta opinião. De fato, a cirurgia bariátrica não está incluída como terapêutica generalizada do DM2, e foi apenas após 2009 que a Associação Americana para Diabetes incluiu a cirurgia bariátrica como alternativa efetiva para o tratamento do DM2 em pacientes com IMC igual ou superior a $35 \mathrm{~kg} / \mathrm{m}^{2} .8,9$

Normalização dos níveis glicêmicos dos pacientes submetidos à cirurgia bariátrica não implica na cura do DM2, porque os níveis glicêmicos podem voltar a estar acima dos limites normais se há reganho de peso. ${ }^{2}$ Há evidências e também muitas questões a serem esclarecidas no tratamento do DM2 mediante procedimentos bariátricos.

\section{Evidências}

\section{Eficácia da cirurgia bariátrica no tratamen- to do DM2}

As cirurgias bariátricas, sobretudo a DGYR e as DBP, têm sido demonstradas eficazes no tratamento do DM2 em pacientes com índice de massa corporal acima de $35 \mathrm{~kg} / \mathrm{m}^{2}$.

\subsection{Em estudos de grandes séries de pacien- tes}

Buchwald et al. publicaram em 2009 os resultados de meta-análise incluindo 135246 pacientes, com valores médios de idade de 40,2 anos e IMC de 47,9 $\mathrm{kg} / \mathrm{m}^{2}$. A perda de excesso de peso média após diferentes tipos de cirurgia bariátrica foi de $55,9 \%$, sendo 63,6\% após DBP, 59,7\% após DGYR e 46,2\% após BGA; paralelamente, a resolução do DM2 foi de $91,1 \%$ após DBP, de 80,3\% após DGYR e de 56,7\% após BGA, com média global de $78.1 \% .{ }^{10}$ Há correlação positiva entre perda de peso pela cirurgia e remissão do DM2.

Sjonstrom et al. demonstraram, em estudo sueco multicêntrico, que nos dois primeiros anos de observação houve resolução completa do DM2 em 72\% de 342 pacientes obesos tratados cirurgicamente e em apenas $21 \%$ dos pacientes do grupo controle. Com 10 anos de seguimento a remissão do DM2 foi também maior nos pacientes submetidos à cirurgia bariátrica $(36 \%)$ que no grupo controle (13\%)..$^{5}$ Alguns dos benefícios observados após a cirurgia podem desaparecer com o passar do tempo, o que pode ser explicado pelo ganho discreto de peso e pelo aumento da idade dos pacientes. ${ }^{11}$

\subsection{Na prevenção de novos casos de diabe- tes}

No estudo multicêntrico sueco, envolvendo grande número de pacientes seguidos em longo prazo, a incidência de novos casos de diabetes nos pacientes avaliados com 2 anos de observação foi de $1 \%$ após cirurgia bariátrica e de $8 \%$ no grupo controle, e com 10 anos de $7 \%$ após cirurgia bariátrica e de $24 \%$ no grupo controle. ${ }^{5}$

\subsection{Na redução do índice de mortalidade re- lacionada ao diabetes após cirurgia ba- riátrica}

A redução da mortalidade com a cirurgia bariátrica tem sido relatada em vários estudos. Adams et al. encontraram, em seguimento de longo prazo, redução significante de óbitos relacionados ao diabetes (92\%) em pacientes submetidos à DGYR. ${ }^{12}$

\section{Mecanismos da cirurgia bariátrica na re- missão do DM2 independentes da perda de peso}

Dois mecanismos principais têm sido apontados para explicar a remissão do DM2 após cirurgias 
bariátricas, independentes da redução calórica e da perda de peso, ambos relacionados à exposição do intestino aos nutrientes.

Uma hipótese relativa aos mecanismos de aumento das incretinas após DGYR é a exclusão do intestino proximal da exposição dos nutrientes inicialmente proposta por Hickey et al. (1998), que observaram que a tolerância à glicose e a sensibilidade à insulina melhoraram após DGYR em comparação com grupo controle. ${ }^{13}$ Rubino e Marescaux demonstraram em ratos diabéticos não-obesos, em comparação com controle (operação simulada), melhora da tolerância à glicose, melhora da sensibilidade à insulina, e diminuição significante da glicemia de jejum, respectivamente, 1,20 e 32 semanas após exclusão do duodeno do trânsito alimentar, mediante derivação duodenojejunal (Figura 4). Em adição, houve um aumento nos níveis de jejum do GLP-1, sem modificação no peso corporal ou na ingestão de alimentos. ${ }^{14}$ Os autores concluem que pode haver um fator presente no intestino proximal, possivelmente uma anti-incretina, que contribui para o perfil do DM2 e que a exclusão dessa porção do intestino delgado pode melhorar o DM2. ${ }^{14}$

Troy et al., em estudo comparativo entre derivação gástrica, que envolve exclusão duodenal, e banda gástrica, em camundongos, encontraram que a exclusão duodenal (ED), além de perda de peso, resultou em redução da ingestão de alimentos e melhora da sensibilidade à insulina; houve aumento da neoglicogênese intestinal apenas após ED. Como mecanismo provável para explicar os efeitos benéficos da exclusão do intestino proximal do trânsito alimentar, relacionados com a ingestão dos alimentos e homeostase da glicose, os autores sugerem uma via sensorial hepatoportal. ${ }^{15}$ (Figura 4)

Uma segunda hipótese para o mecanismo de aumento das incretinas após DGYR e DBP é a rápida exposição do intestino delgado distal aos nutrientes. Patriti et al., em estudo com ratos Goto-Kakisaki, não obesos diabéticos, demostraram que a tolerância à glicose melhorou durante GTT oral 30 dias após transposição ileal (TI), e aumento da sensibilidade à insulina cinco meses após a cirurgia. ${ }^{16}$ Esta operação recoloca segmento de íleo no intestino proximal e permite avaliar o efeito da rápida exposição ao íleo, sem qualquer restrição gástrica ou reorientação do trânsito alimentar. Dessa maneira a TI é capaz de promover um aumento pós-prandial significante nos níveis de GLP-1 e PYY, favorecendo a saciedade e levando ao aumento da secreção de insulina e melhora no metabolismo da glicose. ${ }^{16}$

Esses resultados sugerem que ambas, ED e TI, melhoram a tolerância à glicose. Esses dois mecanismos podem ser os princípios para a remissão do DM2 após DGYR. Entretanto, esses efeitos na tolerância à glicose podem ser mediados por mecanismos diferen-

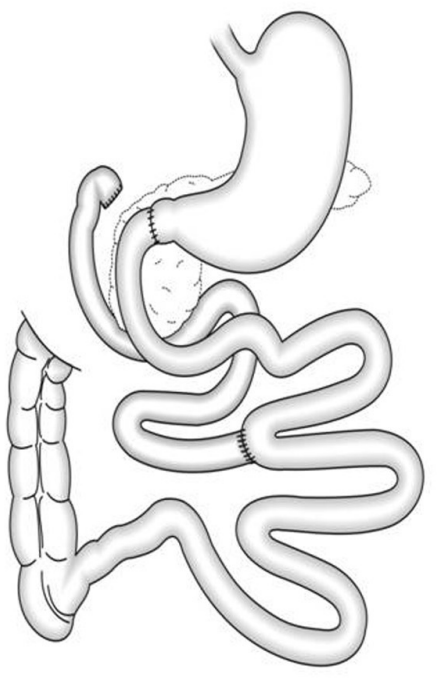

A

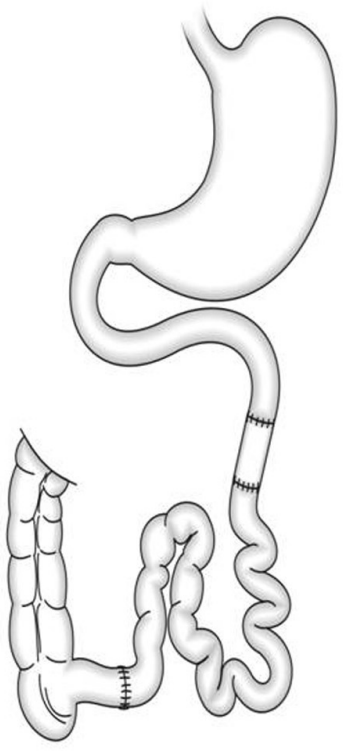

B

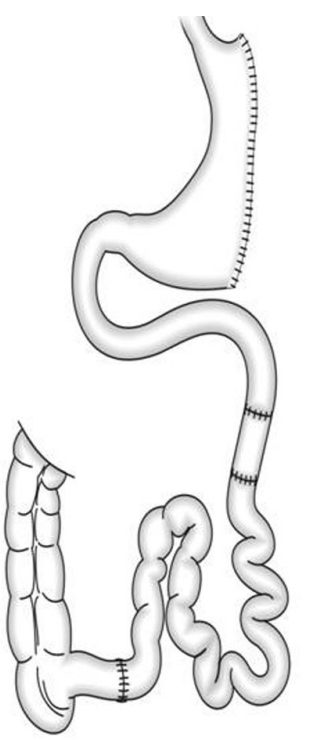

C

Figura 4. Técnicas novas de cirurgia metabólica. A) Exclusão duodenal. B) Interposição ileal. C) Gastrectomia vertical e interposição ileal 
tes. A rápida exposição do intestino distal aos nutrientes pode estar relacionada aos níveis aumentados de GLP-1 após a cirurgia, enquanto a ED pode mediar alguns dos efeitos independentemente de alterações nos níveis de GPL-1.

Há, ainda, relatos de nenhum efeito da ED ou da chegada rápida dos alimentos no intestino distal após DGYR nas alterações nos níveis de GLP-1, a despeito da melhora na tolerância da glicose. Pacheco et al. encontraram que a ED em ratos, similar ao procedimento de Rubino, reduziu os níveis de glicose de jejum, mas não houve qualquer efeito nos níveis de GLP-1 ou de GIP; houve, entretanto, uma redução significante nos níveis de glucagon e de leptina após carga de glicose uma semana após a cirurgia. ${ }^{17}$ Os autores referem que a rápida melhora na homeostase da glicose observada após ED possa ser mediada pela redução nos níveis de leptina, que pode estimular a secreção de insulina, embora nenhuma mudança nos níveis de insulina estimulada pela glicose tenha sido observada após cirurgia.
Alterações pós-operatórias na gliconeogênese intestinal e na microbiota intestinal também foram sugeridas como co-participantes na melhora do DM2 após cirurgias bariátricas. Alterações na microbiota intestinal que podem ocorrer após cirurgia bariátrica poderiam relacionar-se com mudanças metabólicas produzidas pela cirurgia. Estas relações merecem novas investigações.

A Figura 5, baseada em organogramas propostos por Ferrannini et al. ${ }^{18}$ e Bose et al. ${ }^{2}$, ilustra os mecanismos potenciais da cirurgia bariátrica/metabólica na remissão do DM2.

A perda de peso tem um efeito significante na prevenção do desenvolvimento do diabetes, na melhora do controle metabólico e na redução da taxa de mortalidade dos pacientes já diabéticos. Os efeitos benéficos da cirurgia bariátrica no controle da resistência insulínica, do DM2, da hipertensão arterial e de outras co-morbidezes parecem ser proporcionais à perda de peso e inversamente proporcionais ao tempo de evolução do diabetes.

\section{CIRURGIA BARIÁTRICA / METABÓLICA}

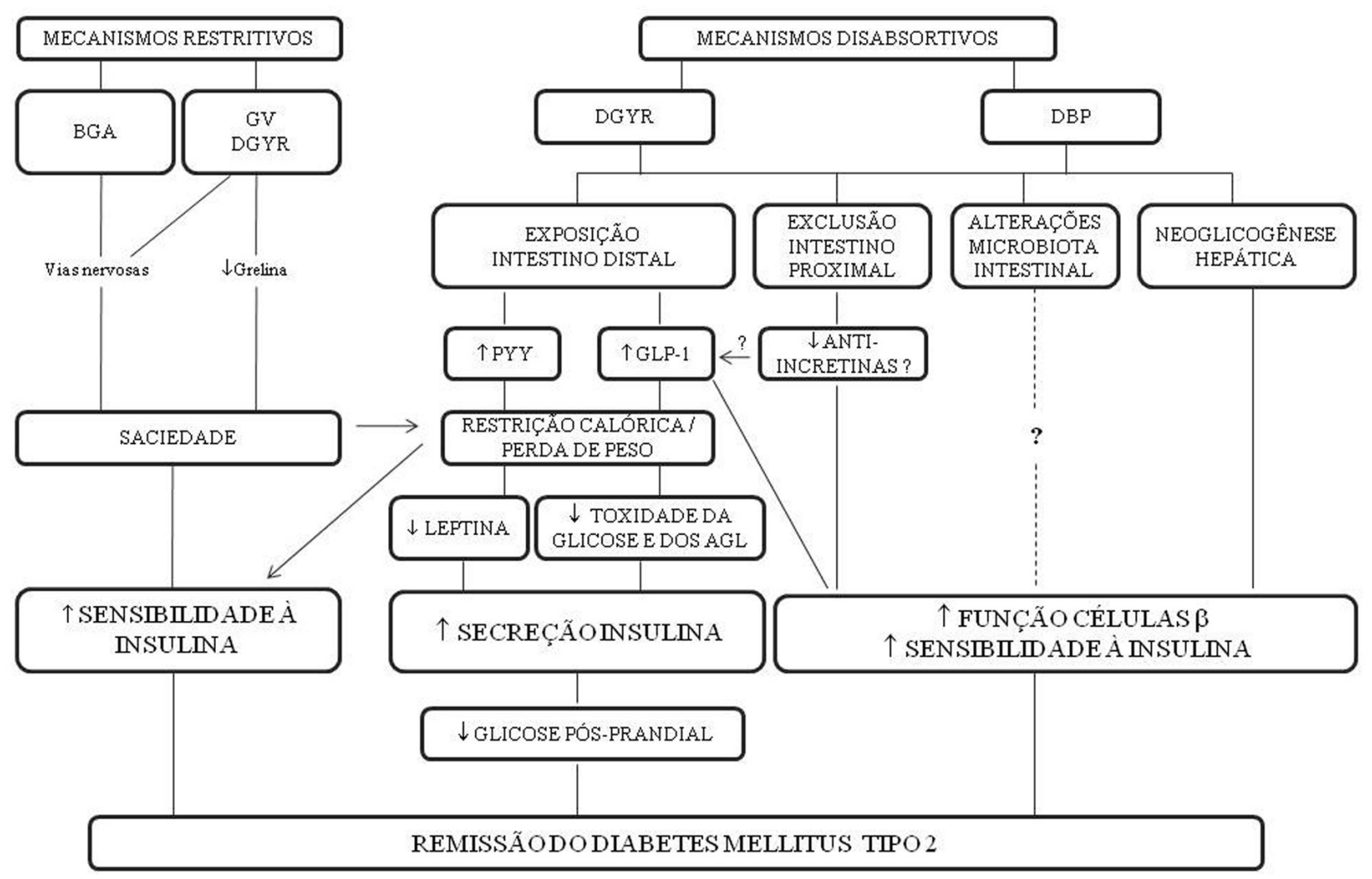

Figura 5. Organograma dos mecanismos potenciais da cirurgia bariátrica/metabólica na remissão do diabetes mellitus tipo 2. 
Os principais achados nos quais se baseiam as hipóteses da presença de mecanismos apontados como independentes da redução da ingestão calórica e perda de peso na remissão do DM2 após cirurgias bariátricas, são os seguintes ${ }^{11}$ :

Rápida melhora do diabetes após cirurgias bariátricas

A melhora do DM2 manifesta-se precocemente, nos primeiros dias a semanas após DGYR e DBP, antes de significante perda de peso. ${ }^{8,19}$

Controle melhor dos níveis glicêmicos e maior secreção de incretina após DGYR do que com tratamento conservador, para perda de peso equivalente

É improvável que a perda de peso contribua para aumentar mais os níveis de incretina do que a natureza cirúrgica da DGYR. Laferrère et al. encontraram, com a mesma perda de peso, liberação de incretina seis vezes maior em pacientes diabéticos submetidos à DGYR do que em pacientes diabéticos submetidos à dieta, o que reforça a ideia de que o efeito incretínico no potencial controle do metabolismo da glicose pode não estar relacionado apenas à perda de peso. ${ }^{20} \mathrm{O}$ efeito do estímulo pós-prandial incretínico persiste por, no mínimo, três anos após DGYR.

Ocorrência de hiperinsulinemia hipoglicêmica mesmo em longo prazo após DGYR decorrente de hiperplasia de ilhotas pancreáticas ou de nesidioblastose. ${ }^{21}$

\section{Dúvidas e contestaçø̃es}

Uma das indicações básicas da cirurgia bariátrica envolve pacientes na faixa etária de 18 a 60 anos com IMC $>35 \mathrm{~kg} / \mathrm{m}^{2}$ na presença de co-morbidezes, incluindo o DM2, independentemente do tempo de duração.

Endocrinologistas, porém, geralmente consideram a cirurgia bariátrica como última opção terapêutica do DM2, somente indicada para pacientes acima de 50 anos de idade portadores de diabetes por mais de 10 anos e com pobre controle metabólico (HbA1c $>7 \%$ ) e, provavelmente, com outras co-morbidezes (hipertensão arterial, dislipidemia aterogênica, esteatohepatite) e complicações microvasculares e macrovasculares, sugerindo que os estudos do tratamento do DM2 devem incluir este tipo de pacientes. ${ }^{9}$ Algumas contestações têm sido feitas sobre a utilização da cirurgia bariátrica no tratamento do DM2 22,23 :

\section{Falta de informações fundamentais em pu- blicações}

A meta-análise de Buchwald et al. ${ }^{7}$ recebe algumas críticas por alguns motivos, tais como falta de informações sobre tempo decorrido desde o início do diabetes, tratamento usado, proporção de pacientes insulinodependentes, grau de controle metabólico dos pacientes, perda de seguimento de parcela significante de pacientes nas séries avaliadas. Também muitos outros trabalhos têm sua qualidade criticada, pelo que autores como Chipkin ${ }^{24}$ e Rubio-Herrera e SanchesPernaute ${ }^{9}$ concluem que não pode ser feita a afirmação geral de que a cirurgia metabólica em pacientes com DM2 com IMC maior que $35 \mathrm{~kg} / \mathrm{m}^{2}$ resulta em alta taxa de resolução do diabetes. A condição clínica prévia é importante para se fazer um juízo realístico da resolução da doença. Apesar dessas críticas, RubioHerrera e Sanches-Pernaute também consideram que a análise geral favorece a cirurgia bariátrica como uma alternativa custo-benefício para o tratamento de pacientes com DM2 com IMC maior que $35 \mathrm{~kg} / \mathrm{m}^{2}$, particularmente se os pacientes selecionados têm pouco tempo desde o início da doença e adequada reserva funcional do pâncreas. ${ }^{9}$ Nesta faixa de peso têm sido realizados os procedimentos cirúrgicos convencionais, sobretudo a DGYR, DBP e BGA, e, mais recentemente também a $\mathrm{GV}$, com o que os pacientes se beneficiam, no mínimo, das vantagens derivadas de uma significante perda de peso, à qual se soma geralmente melhora ou resolução de uma ou mais comorbidezes.

\section{As cirurgias bariátricas não curam o diabe- tes}

Em muitas publicações a remissão do diabetes tem sido interpretada como melhora do DM2 se há normalização da glicemia e redução da dose de drogas hipoglicemiantes ou cura do DM2 se há normalização e interrupção do uso de drogas hipoglicemiantes. É preferível o termo remissão quando não há mais uso de qualquer terapêutica farmacológica ativa, remissão parcial se há hiperglicemia abaixo dos limites diagnósticos de diabetes e remissão completa se glicemia normal. Remissão prolongada corresponde à remissão completa com duração mínima de 1 ano. ${ }^{25}$

Normalização dos níveis glicêmicos dos pacientes submetidos à cirurgia bariátrica não implica na cura do DM2, porque os níveis glicêmicos podem voltar a estar acima dos limites normais se há reganho de peso. 


\section{Mecanismos de ação da cirurgia bariátrica na remissão do DM2}

A perda de peso é fator incontestável na remissão do diabetes de pacientes obesos, o que tem sido comprovado em inúmeros relatos, incluindo a metaanálise de Buchwald et al. ${ }^{10}$ Em adição à perda de peso, a restrição calórica e alterações na secreção de hormônios gastrointestinais podem contribuir para o controle do diabetes.

A melhora do DM2 nos primeiros dias que se seguem à cirurgia bariátrica, quando ainda não há perda de peso significante, tem sido atribuída sobretudo a alterações hormonais, relacionadas principalmente ao aumento das incretinas, e supostamente dependentes da exclusão do duodeno e/ou da chegada rápida dos nutrientes no intestino distal. Aumento pós-prandial do GLP-1 foi bem demonstrado após DGYR, mas o impacto na secreção do GIP é menos consistente. Por outro lado, a BGA, que também favorece o controle da homeostase da glicose e o aumento da sensibilidade à insulina, não envolve derivação intestinal e não resulta no aumento de incretinas, mas induz restrição calórica e perda de peso. A grelina, hormônio com efeito orexígeno, também exerce modulação no metabolismo da glicose. A secreção de grelina aumenta com a perda de peso não dependente de cirurgia bariátrica e diminui nos obesos e após DGYR.

Os mecanismos da melhora precoce do DM2 após cirurgia bariátrica, porém, permanecem incertos.

Alguns relatos contestam a importância dos hormônios gastrointestinais na melhora precoce do DM2 após cirurgias bariátricas.

Morinigo et al. demonstraram, em estudo comparativo entre obesos diabéticos e não diabéticos, diminuição da glicemia e da HbA1c e melhora da sensibilidade à insulina 6 semanas após DGYR nos dois grupos de pacientes; entretanto, houve aumento do GLP-1 apenas nos pacientes não diabéticos, sugerindo que o GLP-1 não é fator crucial para a melhora pós-operatória precoce da tolerância à glicose. ${ }^{26}$

Isbell et al. demonstraram, em estudo prospectivo controlado, que a restrição calórica, sem perda de peso significante, é fator de importância fundamental na melhora da sensibilidade insulínica na primeira semana (4 dias em média) após DGYR, sem qualquer benefício adicional do aumento do GLP-1 no controle da glicemia. ${ }^{27}$

\section{Cirurgia metabólica para pacientes com IMC $<35 \mathrm{~kg} / \mathrm{m}^{2}$}

Nesses pacientes a cirurgia bariátrica, diferentemente que para os obesos mórbidos, é considerada uma estratégia inicial para o tratamento do DM2, mais do que uma alternativa para perda de peso.

Há grande controvérsia em relação ao tratamento cirúrgico para esses pacientes. A aceitação da cirurgia metabólica seria mais fácil para os pacientes com co-morbidezes outras, DM2 de controle difícil e risco cardíaco significante. O problema maior está no grande grupo de pacientes diabéticos sem co-morbidezes e que estão bem controlados com hipoglicemiantes orais, provavelmente os que mais se beneficiariam com a cirurgia metabólica, já que a experiência com obesos mórbidos mostra que os piores resultados são encontrados em pacientes insulinodependentes e DM2 de longa duração.

Na ausência de consenso, há uma consideração importante na qual a maioria dos profissionais concorda: deve existir reserva pancreática adequada (peptídeo C $>1 \mathrm{ng} / \mathrm{dl}$ ). É indispensável também eliminar a possibilidade de diabetes autoimune em adultos, que acomete até $10 \%$ dos pacientes com DM2 assumido. ${ }^{28}$

Pesquisadores, como Cohen, julgam que os valores do IMC utilizados para definir indicação de tratamento cirúrgico são parâmetros arbitrários que podem retardar uma opção potencialmente salvadora da vida para pacientes com IMC menor que $35 \mathrm{~kg} / \mathrm{m}^{2}$. ${ }^{29}$

Reforçam essa ideia os bons resultados da DGYR no tratamento de pacientes com DM2 com $\mathrm{IMC}<35 \mathrm{~kg} / \mathrm{m}^{2}$ relatados por Cohen em $2006^{30}$ e por Chiellini em 2009. ${ }^{31}$ Sabe-se que nem todos os obesos mórbidos têm problemas metabólicos e há mesmo aproximadamente $20 \%$ de pacientes com obesidade grau III que não têm RI (obesos metabolicamente saudáveis). Há evidência cumulativa que identifica a inflamação crônica como a ligação entre acúmulo de tecido adiposo e complicações cardíacas e metabólicas. ${ }^{32}$ Seria, assim, interessante priorizar pacientes que têm problemas metabólicos e que mais se beneficiariam com a cirurgia bariátrica.

\section{Seleção dos pacientes para cirurgia meta- bólica}

Parece que a cirurgia metabólica, como o tratamento medicamentoso, se ajusta melhor se baseada 
na história natural do DM2. A função preservada das células $\beta$ é essencial para o efeito das incretinas na secreção de insulina.

Parece não razoável a indicação de cirurgia metabólica para pacientes com DM2 no estágio final, pois as incretinas necessitam da integridade das células $\beta$ para exercer sua função hipoglicemiante. Também não parece adequada a cirurgia metabólica para o estágio precoce do diabetes, quando hábitos saudáveis, modificações na dieta e antidiabéticos orais são efetivos e quase sem risco. O tempo ideal para considerar a cirurgia metabólica como opção de tratamento do DM2 talvez seja o estágio intermediário da doença, quando os antidiabéticos orais começam a falhar e há alto grau de RI e há massa de células $\beta$ ainda preservada que pode maximizar o efeito dos peptídeos intestinais que aumentam a secreção de insulina. ${ }^{25}$

Embora a perda de peso seja uma garantia para melhora da qualidade de vida e da sensibilidade à insulina, existem fatores de risco para recorrência do DM2 em longo prazo após cirurgia bariátrica ${ }^{9}$ :

- Tempo decorrido do início do DM2 > 10 anos

- Tratamento com insulina

- Controle metabólico pobre (HbA1c > 8\%)

- Perda de excesso de peso $<50 \%$

- Peptídeo $\mathrm{C}<1$ ng/dl

Na seleção dos pacientes, além da necessidade imperiosa de se considerar duração e gravidade do DM2 (idade do paciente, presença de alterações metabólicas, resposta ao tratamento conservador e capacidade funcional das células $\beta$ ), é importante eliminar a possibilidade de se tratar de diabetes autoimune em adulto não assumida e ter em mente que os melhores resultados da cirurgia metabólica têm correlação positiva com o grau de redução ponderal no pósoperatório e correlação negativa com o valor do IMC pré-operatório.

\section{Tipo de procedimento cirúrgico}

A cirurgia bariátrica é um meio efetivo de induzir a remissão do diabetes em pacientes muito obesos com DM2.

A ordem crescente de eficácia dos procedimentos bariátricos mais comuns progride dos puramente restritivos aos mistos predominantemente restritivos e depois aos predominantemente disabsortivos. São procedimentos que visam, mediante perda de peso, prevenir ou tratar co-morbidezes da obesidade.
Cohen et al., relataram os resultados da DGYR em 37 pacientes com DM2 tratados com hipoglicemiantes orais, com IMC médio de $32,5(32-34,9) \mathrm{kg} / \mathrm{m}^{2}$ e seguidos por 6 a 48 meses; o tratamento cirúrgico resultou em perda de $81 \%$ do excesso de peso e resolução do diabetes em todos os pacientes. ${ }^{29}$

Os bons resultados da cirurgia convencional na resolução e melhora do DM2, sobretudo por serem evidentes precocemente no pós-operatório, antes de perda significativa de peso, e os resultados animadores de pesquisas em animais motivaram investigações sobre novas abordagens cirúrgicas em pacientes diabéticos com IMC inferior aos utilizados como indicação de cirurgia bariátrica.

Cohen et al., em 2007, relataram os resultados da derivação duodenojejunal em dois pacientes com IMC de 27 e $29 \mathrm{~kg} / \mathrm{m}^{2}$ e DM2 de duração de 2 e 7 anos, respectivamente sob tratamento com insulina e metformina. Aos 9 meses de seguimento encontraram resolução do DM2 em ambos pacientes, sem medicamentos e com HB1Ac de 5 e 5,7 \% e IMC de 27 e $29,5 \mathrm{~kg} / \mathrm{m}^{2}$, respectivamente. ${ }^{29}$

Geloneze et al., em 2009, em 12 pacientes com DM2 e IMC entre 25 e $29,9 \mathrm{~kg} / \mathrm{m}^{2}$, submetidos à ED e seguidos por 24 semanas em comparação com grupo controle de pacientes diabéticos em condições semelhantes, não operados e sob tratamento padrão, observaram nos pacientes operados maior redução na glicemia de jejum (14\% versus $7 \%$ ), maior redução da $\mathrm{HbA1c}(8,78$ para 7,84 versus 8.93 para 8,71$)$ e redução no requerimento diário de insulina ( $93 \%$ versus $29 \%$ ). Não houve diferença entre os grupos quanto ao IMC, composição corpórea, pressão sanguínea e lípides. ${ }^{33}$

De Paula et al., em 2008, examinaram os efeitos clínicos da TI em associação com GV, ou com GV mais derivação duodenal, em 39 pacientes com DM2 e IMC médio de $30,1 \mathrm{~kg} / \mathrm{m}^{2}$, sendo que a maioria estava usando hipoglicemiante por via oral. Sete meses após a cirurgia os pacientes perderam em média $22 \%$ de seu peso pré-operatório (IMC $=24,9 \mathrm{~kg} / \mathrm{m}^{2}$ ) e apresentavam redução significante da HbA1c (28\%), da glicose de jejum (44,6\%) e pós-prandial $(45,3 \%)$ e da HOMA-IR (50\%). Os níveis de incretina não foram relatados. História de diabetes menor que 5 anos e IMC entre 30 e $35 \mathrm{~kg} / \mathrm{m}^{2}$ foram fatores associados a controle metabólico melhor; melhora significante também foi demonstrada para hipertensão arterial, dislipidemia, função renal e retinopatia diabética. A mortali- 
dade operatória foi de 2,6\%. Complicações pós-operatórias maiores aconteceram em $10,3 \%$ dos pacientes, incluindo fístulas gástricas em 2 pacientes. ${ }^{34}$ Esta cirurgia parece induzir a uma melhora importante no controle do diabetes e de suas co-morbidezes, porém sua complexidade é responsável por alta taxa de mortalidade e de complicações. ${ }^{35}$

Recentes desenvolvimentos em cirurgia bariátrica experimental tais como exclusão duodenal, transposição ileal e gastrectomia vertical requerem estudos mais cuidadosos antes de se tornarem aplicáveis clinicamente em larga escala. É importante conhecer melhor como esses tratamentos melhoram condições metabólicas como o DM2. ${ }^{2}$

Os resultados de muitos estudos citados sugerem que as incretinas, especialmente o GLP-1, participam dos efeitos benéficos da cirurgia bariátrica no controle metabólico dos pacientes com DM2. Os mecanismos pelos quais esta participação acontece ainda permanecem obscuros. Muitos aspectos necessitam ser elucidados. Os efeitos da GV na resposta do GLP-1 pós-prandial sugerem que a exclusão duodenal não é necessária para estimular a produção de incretina, embora o controle glicêmico da GV seja menos evidente que da DGYR e da DBP com duodenal switch. Por outro lado, os resultados da derivação duodenojejunal mostram que esta modificação da anatomia intestinal também pode participar do controle da hiperglicemia. ${ }^{35}$

Pode-se especular que exclusão do intestino proximal e chegada rápida dos nutrientes no intestino distal participam da homeostase da glicose e, por isso, os benefícios para os pacientes com DM2 sejam mais evidentes após DGYR e DBP, justamente os procedimentos que incorporam esses dois mecanismos. Outros hormônios gastrointestinais necessitam mais estudos. Os critérios de resolução ou melhora do diabetes podem ser questionáveis, mas os efeitos da exclusão duodenal e da transposição ileal no controle metabólico do DM2 abrem novas perspectivas no campo de cirurgia metabólica. ${ }^{35}$

Apesar das pesquisas disponíveis, há significantes limitações em muitos estudos. As séries de pacientes com IMC $<35 \mathrm{~kg} / \mathrm{m}^{2}$ submetidos à cirurgia metabólica são pequenas e o tempo de seguimento é pequeno. Grande número de estudos sobre alterações hormonais relacionadas à homeostase da glicose após cirurgia têm sido conduzidos em pacientes não diabéticos. Fatores como história familiar, interação com terapias antidiabéticas prévias ou evidência de auto- imunidade nem sempre têm sido analisadas especificamente. Duração e estado do diabetes, resposta ao tratamento medicamentoso, parâmetros bioquímicos que distinguem claramente a eventual presença do diabetes tipo1, avaliação da capacidade funcional das células $\beta$ e critérios adequados para caracterizar resolução do diabetes, entre outros fatores, devem fazer parte das pesquisas sobre cirurgia metabólica. Ainda, perda de peso e outros fatores devem ser considerados, principalmente em longo prazo.

O estado atual da cirurgia metabólica no tratamento do DM2 está evidente nas recomendações da Associação Americana para o Diabetes (ADA) de 2010:

1. A cirurgia bariátrica deve ser considerada para adultos com IMC igual ou maior que $35 \mathrm{~kg} / \mathrm{m}^{2}$ e DM2, especialmente se o diabetes ou co-morbidezes presentes são de difícil controle com modificação do estilo de vida e terapêutica farmacológica.

2. Embora experiências com séries pequenas tenham mostrado benefício glicêmico da cirurgia bariátrica em pacientes com DM 2 e IMC entre 30 e $35 \mathrm{~kg} / \mathrm{m}^{2}$ há atualmente evidência insuficiente para recomendar cirurgia em larga escala para pacientes com IMC menor que $35 \mathrm{~kg} / \mathrm{m}^{2}$ fora de protocolo de pesquisa.

3. Benefícios em longo prazo, custos e vantagens, e riscos da cirurgia bariátrica em pacientes com DM2 deverão ser estudados em pesquisas bem planejadas, controladas e casualizadas tendo como controle grupos sob terapêutica médica e estilo de vida corretos.

\section{Conclusões}

1. A cirurgia bariátrica tem sido aceita como opção terapêutica efetiva para pacientes com diabetes mellitus tipo 2 e índice de massa corporal maior que $35 \mathrm{~kg} / \mathrm{m}^{2}$, em particular quando a duração do diabetes é curta e há ainda boa capacidade funcional das células $\beta$ do pâncreas.

2. Os melhores resultados têm sido obtidos para índices de massa corporal maiores e com os procedimentos cirúrgicos que conseguem maior redução de peso.

3. Não há atualmente evidência suficiente para recomendar cirurgia em larga escala para pacientes com diabetes tipo 2 com índice de massa corporal menor que $35 \mathrm{~kg} / \mathrm{m}^{2}$, mas há evidências preliminares para suportar a continuação de investigação da ci- 
rurgia metabólica como meio possível de conseguir resolução duradoura do DM2 na vasta população de obesos não mórbidos; para esses pacientes a cirurgia metabólica somente está justificada como parte de protocolos de pesquisa bem elaborados e aprovados pela Comissão de Ética em Pesquisa do hospital e pelo Comitê Nacional de Ética em Pesquisa.

4. Novas pesquisas de boa qualidade e controladas são recomendadas, focalizando sobretudo: mecanismos fisiopatológicos que possam explicar os efeitos das cirurgias bariátricas no metabolismo da glicose, em particular os que participam da remissão do diabetes; desenvolvimento de novos procedimentos cirúrgicos especialmente ajustados para o controle do diabetes tipo 2; e os possíveis efeitos da cirurgia metabólica nos diabéticos tipo $2 \mathrm{com}$ índice de massa corporal menor que $35 \mathrm{~kg} / \mathrm{m}^{2}$, em séries bem definidas de pacientes, de acordo com idade, sexo, etnia e duração do diabetes.

\section{Referências bibliográficas}

1. Albert KG, Zimmet P, Shaw J. International Diabetes Federation: a consensus on type 2 diabetes prevention. Diabet Med. 2007; 24:451-63.

2. Bose $M$, Oliván B, Teixeira J, Pi-Sunyer FX, Laferrère B. Do incretins play a role in the remisson of type 2 diabetes after gastric bypass surgery: what are the evidences? Obes Surg, 2009; 19:217-29.

3. Gastaldelli A, Ferrannini E, Miyzaki Y, Matsuda M, DeFronzo RA. San Antonio metabolism Study. Beta-cell disfunction and glucose intolerance results from the San Antonio metabolism (SAM) study. Diabetologia. 2004; 47:31-9.

4. Hu FB, Manson JE, Stampfer MJ, Colditz G, Liu S, Solomon CG, et al. Diet, lifestyle, and the risk of type 2 diabetes mellitus in women. New Engl J Med. 2001; 345:790-7.

5. Sjostrom L, Lindroos AK, Peltonen M, Torgerson J, Bouchard C, Carlsson B, et al. Swedish Obese Subjects Study Cientific Group. Lifestyle, diabetes, and cardiovascular risk factors 10 years after bariatric life. N Engl J Med. 2004; 351:2683-93.

6. Pories WJ, Swanson MS, MacDonald KG, Long SB, Morris PG, Brown BM, et al. Who would have thought it? An operation proves to be the most effective therapy for adult-onset diabetes mellitus. Ann Surg. 1995; 222:339-50.

7. Buchwald $H$, Avidor $Y$, Braunwold $E$, Jensen $M$, Pories WJ, Fahrbach $\mathrm{K}$ et al. Bariatric surgery: a systematic review and meta-analysis. JAMA. 2004; 292:1724-37.

8. Rubino F, Kaplan IM, Schauer PR, Cummings DE. Diabetes Surgery Summit Delegates. The Diabetes Surgery Summit consensus conference: recommendations for the evaluation and use of gastrointestinal surgery to treat type 2 diabetes mellitus. Ann Surg. 2010; 251:399-405.

9. Rubio-Herrera MA, Sanchez-Pernaute A. Indications of surgery for type 2 diabetes. Debate: An against position. In: GarciaCaballero M, Tinahones FJ, Cohen RV, editors. Diabetes surgery. fst ed. Madrid: McGraw Hill; 2010. p. 301-12.
10. Buchwald H, Estok R, Fahrbach K, Banel D, Jensen MD, Poories WJ, et al. Weight and type 2 diabetes after bariatric surgery: systematic review and meta-analysis. Am J Med. 2009; 122:248-56

11. Busetto L, Sbraccia P, Frittitta L, Pontiroli AE. The growing role of bariatric surgery in the management of type 2 diabetes: evidences and open questions. Obes Surg. 2011; 21:1451-7.

12. Adams TD, Gress RE, Smith SC, Halverson RC, Simper SC, Rosamond WD. et al. Long-term mortality after gastric bypass surgery. N Engl J Med. 2007; 357:753-61.

13. Hickey MS, Pories WJ, MacDonald KG Jr, Cory KA, Dohm GL, Swanson MS, et al. A new paradigm for type 2 diabetes mellitus: could it be a disease of the foregut? Ann Surg 1998; 227:637-43. discussion 43-4.

14. Rubino F, Marescaux J. Effect of duodenal-jejunal exclusion in a non-obese animal model of type 2 diabetes: a new perspective for an old disease. Ann Surg Rubino F, Marescaux $\mathrm{J}$. Effect of duodenal-jejunal exclusion in a non-obese animal model of type 2 diabetes: a new perspective for an old disease. Ann Surg. 2004; 239:1-11.

15. Troy S, Soty M, Ribeiro L, Laval L, Migrenne S, Fioramonti X, et al. Cell Metab 2008; 8:201-11.

16. Patriti A, Facchiano E, Annetti C, Alsa MC, Galil F, Fanelli C, et al. Early improvement of glucose tolerance after ileal transposition in a non-obese type 2 diabetes rat model. Obes Surg. 2005; 15:1258-64.

17. Pacheco D, de Luis DA, Romero A, Sagrado MG, Conde R, Izaola $\mathrm{O}$, et al. The effects of duodenal-jejunal exclusion on hormonal regulation of glucose metabolism in Goto-Kakizaki rats. Am J Surg. 2007; 194:221-4.

18. Ferraninni L, Mingroni G. Impact of different bariatric surgical procedures on insulin action and $\beta$ cell function in type 2 diabetes. Diabetes Care. 2009; 32:514-20.

19. Schauer PR, Bruguera B, Ikramuddin S, Cottam D, Gourash W, Hamad G, et al. Effect of laparoscopic Roux-en-Y gastric bypass on type 2 diabetes mellitus. Ann Surg. 2003; 238:46784.

20. Laferrère B, Teixeira J, McGinty J, Tran H, Egger JR, Colarusso $A$, et al. Effect of weight loss by gastric bypass surgery versus hypocaloric diet on glucose and incretin levels in patients with type 2 diabetes. J Clin Endocrinol Metab. 2008; 93: 2479-85.

21. Service GJ, Thompson GB, Service FJ, Andrews JC, CollazoClavell MI, Ricardo V. Hyperinsulinemic hypoglycemia with nesioidioblastosis after gastric bypass surgery. N Engl J Med. 2005; 353: 249-54.

22. Schernthaner G, Kopp HP, Brix JM, Schernthaner GH. Cure of type 2 diabetes by metabolic surgery? A critical analysis of the evidence in 2010. Diabetes Care. 2011; 34:S5355-60.

23. Pinkney JH, Johnson AB, Gale EA. Diabetologia. 2010; 53:1815-22.

24. Chipkin SR, Godberg RJ. Obesity surgery and diabetes: does a chance to cut mean to cure? Am J Med, 2009; 122:205-6.

25. Buse JB, Caprio S, Cefalu WT, Ceriello A, Del Prato S, Inzucchi S. How do we define cure of diabetes? Diabetes Care. 2009; 32:2133-5.

26. Morinigo R, Lacy AM, Casamitjana R, Delgado S, Gomis R, Vidal J. GLP-1 and changes in glicose tolerance following gastric by-pass surgery in morbidly obese patients. Obes Surg. 2006; 16:1594-601.

27. Isbell JM, Tamboli RA, Hansen EN, Saliba J, Dunn JP, Phillips $\mathrm{SE}$, et al. The importance in caloric restriction in the early 
improvements in insulin sensitivity followung Roux-en-Y gastric bypass surgery. Diabetes Care. 2010; 33:1438-47.

28. Zimmet P, Turner R, McCarty D, Rowley M, Mackay I. Crucia points at diagnosis. Type 2 diabetes or slow type 1 diabetes. Diabetes Care. 1999; 22:B59-64.

29. Cohen RV, Schiavon CA, Pinheiro JS, Correa JL, Rubino F. Duodenal-jejunal bypass for the treatment of type 2 diabetes patients with body mass index of $22-34 \mathrm{~kg} / \mathrm{m}^{2}$ : a report of 2 cases. Surg Obes Relat Dis. 2007; 3:195-7.

30. Cohen RV, Pinheiro JS, Correa JL, Schiavon CA. Laparoscopic Roux-en-Y gastric bypass for $B M \mid<35 \mathrm{~kg} / \mathrm{m}^{2}$; a tailored approach. Surg Obes Relat Dis. 2006; 2:401-4.

31. Chiellini C. The effect of bilio-pancreatic diversion on type 2 diabetes in patients with $\mathrm{BMl}<35 \mathrm{~kg} / \mathrm{m}^{2}$. Diabetología. 2009; 52:1027-30.

32. Murri M, Tinahones FJ. Intestinal changes other than incretins. In: Garcia-Caballero M, Tinahones FJ, Cohen RV, editors. Diabetes surgery. fst ed. Madrid: McGraw Hill; 2010. p. 34755.
33. Geloneze B, Geloneze SR, Fiori C, Stabe C, Tambascia MA Chaim EA, et al. Surgery for nonobese type 2 diabetic patients: an interventional study with duodenal-jejunal exclusion. Obes Surg. 2009; 19:1077-83.

34. De Paula AL, Macedo AL, Rassi N, Machado CA, Schraibman V, Silva LQ, et al. Laparoscopic treatment of type 2 diabetes mellitus for patients with body mass index less than 35 . Surg Endosc 2008; 22:706-16.

35. Salvador J, de la Higuera M, Escalada J, Silva C, Rotellar F, Gomes-Ambrosi Salvador J, et al. Role of incretins in the beneficial effects of bariatric and metabolic surgery on type 2 diabetes mellitus. In: Garcia-Caballero M, Tinahones FJ, Cohen RV, editors. Diabetes surgery. 1st ed. Madrid: McGraw Hill; 2010. p. 339-45. 